\title{
Anemia y adherencia a la suplementación oral con hierro en una muestra de niños usuarios de la red de salud pública de Rosario, Santa Fe Anemia and compliance to oral iron supplementation in a sample of children attending the public health network of Rosario, Santa Fe
}

Dr. Logan Christensen ${ }^{a}$, Dra. Yanina Sguassero ${ }^{b}$ y Lic. Cristina B. Cuesta ${ }^{b}$

\section{RESUMEN}

Introducción. La suplementación con hierro medicinal es una intervención gratuita y ampliamente utilizada para prevenir y tratar la anemia infantil.

Objetivos. Determinar la prevalencia de la anemia en una muestra de niños rosarinos, describir el uso de suplementos con hierro en los niños de la muestra estudiada y detallar las variables potencialmente relacionadas con la adherencia de las madres a la administración oral del hierro.

Población y métodos. Se realizó un estudio transversal que incluyó a madres y niños menores de 42 meses atendidos en la red de salud pública de la ciudad de Rosario entre diciembre de 2011 y abril de 2012. Se recolectaron variables sociodemográficas y datos sobre salud, crecimiento, anemia y administración de hierro en el niño. Se usó una prueba rápida para determinar el nivel de hemoglobina.

Resultados. Se incluyeron 325 pares de madres y niños. La prevalencia global de la anemia fue del $40 \%$ (IC $95 \% 35 \%$ a $45 \%$ ) y aumentó hasta un $56 \%$ en el grupo de 6 a 23 meses. El 51\% de las madres refirieron que su hijo había tomado hierro alguna vez. La adherencia de las madres a la administración del hierro fue mayor en los niños sin anemia en comparación con los niños con anemia (OR0,28;IC 95\% 0,1 a 0,69). Las causas más frecuentes de falta de adherencia fueron la intolerancia digestiva ( $38 \%$ ) y el olvido $(36 \%)$. Conclusiones. La prevalencia de la anemia infantil en la muestra estudiada fue alta. Se observó menor adherencia de las madres a la administración del hierro en el grupo de niños con anemia.

Palabras clave: anemia, lactantes, hierro, administración oral, cumplimiento de la medicación.

Global Health Institute, Field Study Grant.

Duke University,

Durham NC, EE.UU.

Correspondencia:

Dra. Yanina Sguassero: ysguassero@crep.org.ar

Conflicto de intereses: Ninguno que declarar.

Recibido: 15-10-2012 Aceptado: 26-2-2013 http://dx.doi.org/10.5546/aap.2013.xx

\section{INTRODUCCIÓN}

Según datos de la OMS sobre 35 países de la región de las Américas, la prevalencia de la anemia en los niños de edad preescolar es del $47 \%$ (alrededor de 23 millones de niños). ${ }^{1}$ Se presume que la mitad de las anemias son causadas por déficit de hierro, la deficiencia nutricional de mayor prevalencia en los países de bajos y medianos ingresos. ${ }^{2}$

La determinación de hemoglobina $(\mathrm{Hb})$ y la de ferritina séricas son dos pruebas de laboratorio comúnmente utilizadas para el diagnóstico de anemia ferropénica. A pesar de que no se conoce el valor a partir del cual la deficiencia de hierro produce efectos perjudiciales, ${ }^{3}$ la OMS recomienda los siguientes valores límites para los niños de 6 a 23 meses de edad: $\mathrm{Hb}$ $<11 \mathrm{~g} / \mathrm{dL}$ y ferritina $<10-12 \mu \mathrm{g} / \mathrm{L} .{ }^{4}$ En los menores de 6 meses no existe consenso para definir anemia; 9,5 $\mathrm{g} / \mathrm{dL}$ es uno de los puntos de corte sugeridos para la $\mathrm{Hb} .^{5}$

La mayoría de los trabajos sobre la prevalencia de la anemia en la región del Cono Sur provienen de Brasil e informan* que es del $30 \%$ al $64 \%{ }^{6-10}$ En nuestro país, los datos más representativos provienen de la Encuesta Nacional de Nutrición y Salud 2005. ${ }^{11}$ Sus resultados mostraron una prevalencia total de anemia (definida como $\mathrm{Hb}<11 \mathrm{~g} / \mathrm{dL}$ ) en los niños de 6 a 60 meses del 16\%, aumentando en el grupo de 6 a 23 meses hasta un $34 \%$.

Las intervenciones tendientes a prevenir la anemia infantil se han agrupado según su impacto a lo largo del tiempo: ${ }^{12}$

\footnotetext{
* Los detalles de la estrategia de búsqueda están disponibles por pedido al autor principal.
} 
- Corto plazo: ligadura tardía del cordón umbilical, ${ }^{13}$ promoción de la lactancia materna exclusiva, introducción oportuna de una adecuada alimentación complementaria y suplementación con hierro medicamentoso.

- Mediano plazo: fortificación de los alimentos con hierro.

- Largo plazo: educación alimentaria y nutricional, campañas de desparasitación, saneamiento ambiental y estrategias de educación comunitaria.

En el año 2009, la Sociedad Argentina de Pediatría publicó una guía nacional sobre prevención y tratamiento de la anemia ferropénica. ${ }^{4}$ Basándose en estas recomendaciones, se debería solicitar pruebas de laboratorio para detectar la anemia a todo niño de entre 9 y 12 meses de edad. Correspondería, además, prescribir sulfato ferroso en dosis profilácticas a los niños con mayor riesgo, como los recién nacidos prematuros, los gemelares y los recién nacidos de término alimentados con leche de vaca o con pecho, pero con una dieta pobre en hierro a partir del sexto mes de vida. En todos los casos, la administración del hierro debería prolongarse hasta los 12 a 18 meses de edad.

El sulfato ferroso es uno de los medicamentos que se provee de forma gratuita a través del Programa Nacional Remediar. ${ }^{15}$ Existen trabajos publicados en nuestro país acerca de los factores que podrían interferir en la eficacia de la suplementación con hierro en las poblaciones

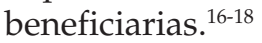

Una revisión publicada por Lutter y cols. ${ }^{19}$ mostró la eficacia de controlar la adherencia a la suplementación con hierro para disminuir la prevalencia de la anemia infantil, mientras que otros estudios ${ }^{20,21}$ sobre estrategias alternativas comunicaron resultados contradictorios.

Los objetivos principales de este estudio son determinar la prevalencia de la anemia en una muestra de niños rosarinos, describir el uso de suplementos con hierro en los niños de la muestra estudiada y detallar las variables potencialmente relacionadas con la adherencia de las madres a la administración oral de hierro.

\section{POBLACIÓN Y MÉTODOS}

En el marco de un proyecto internacional sobre el desarrollo infantil temprano, ${ }^{22}$ se realizó un estudio descriptivo de corte transversal en la red de atención pública de la ciudad de Rosario. La selección de los centros de salud se basó en la disponibilidad de un médico pediatra que tuviera interés en colaborar con el proyecto y de un espacio físico adecuado para entrevistar a las madres. Cinco centros participaron en el estudio: Hospital de Niños V. J. Vilela, Maternidad Martin, Centro de Atención Primaria Eva Duarte, Hospital J. B. Alberdi y Hospital Escuela Eva Perón. La muestra del estudio se seleccionó a partir de la población de referencia de los centros participantes, e incluyó a madres y niños menores de 42 meses que fueron atendidos en el Servicio de Pediatría ambulatoria durante el período comprendido entre diciembre de 2011 y abril de 2012.

Los criterios de exclusión locales que se aplicaron en el momento de invitar a las madres a participar en el estudio fueron: diagnóstico de enfermedades genéticas o neurológicas, cuadros infecciosos y fiebre.

Los datos fueron recabados por investigadores entrenados para las tareas asignadas. Las variables de interés consideradas para este estudio fueron: edad (años), estado civil de la madre, nivel de educación de la madre (años), características de la vivienda (agua corriente, baño con botón o cadena, paredes de cemento o ladrillo, electricidad, heladera, televisión), otorgamiento de subsidio para el niño (sí/no), peso al nacer (gramos), duración de la lactancia materna (meses), antecedentes de desnutrición, anemia y administración de hierro en el niño (sí/no).

Si la madre refería que el niño había tomado hierro alguna vez, se solicitaba la siguiente información adicional: nombre del preparado con hierro, edad (meses) al comienzo de la administración del suplemento, ingesta actual del suplemento con hierro (sí/no), edad (meses) al final de la suplementación.

La adherencia de las madres se estudió en el grupo de niños que estaban tomando hierro durante al menos un mes o habían dejado de tomarlo dentro de los 6 meses anteriores al día de la entrevista. En este grupo de madres, se recolectó información complementaria a través de la siguiente pregunta abierta: ¿Cómo le ha dado el hierro al niño la semana pasada? Luego se le preguntaba a la madre si eso había sido indicado por el médico.

Si la madre refería que el niño nunca había tomado hierro y en los casos de falta de adherencia, se le preguntaba de forma abierta sobre el motivo por el cual no lo había hecho.

Se midió la concentración de $\mathrm{Hb}$ con un hemoglobinómetro portátil ${ }^{23}$ a partir de una gota 
de sangre del dedo índice del niño. Se entregó el resultado de la prueba al médico pediatra y se anotó el peso $(\mathrm{kg})$, la longitud/talla $(\mathrm{cm})$ y el perímetro cefálico $(\mathrm{cm})$ del niño obtenidos durante la consulta clínica.

Se consideraron las siguientes definiciones:

- Lactancia materna exclusiva: lactante alimentado con leche materna (incluida leche materna extraída o de nodriza) y que además puede recibir gotas, jarabes (vitaminas, minerales, medicinas) durante los primeros 6 meses de vida.

- Bajo peso: peso para la edad por debajo de -2 desviaciones estándar (DE) de la referencia actualmente en uso en la Argentina.

- Baja talla: longitud/talla por debajo de -2 DE de la referencia actualmente en uso en la Argentina.

- Emaciación: peso para la longitud/talla por debajo de -2 DE de la referencia actualmente en uso en la Argentina.

- Anemia: hemoglobina sérica por debajo de $11 \mathrm{~g} / \mathrm{dL}$ en los niños de 6 meses o más ${ }^{4,11,12}$ y por debajo de $9,5 \mathrm{~g} / \mathrm{dL}$ en los $<6$ meses. ${ }^{5}$

- Adherencia: administración oral de hierro $\geq 5$ días/semana y según indicación médica.

- Falta de adherencia: administración oral de hierro $<5$ días/semana o suspensión del tratamiento sin respetar la indicación médica.

El análisis de los datos se realizó de forma global y para los siguientes grupos de edades: niños menores de 6 , de 6 a 23 y $\geq 24$ meses. Se utilizó el programa SAS 9.2 para realizar los análisis estadísticos. ${ }^{24}$ Las variables categóricas se describieron como porcentajes. Se calculó, además, el intervalo de confianza (IC) del 95\% para los resultados principales. Las variables continuas se describieron como media y desviación estándar. Las comparaciones entre los grupos del estudio se realizaron con la prueba exacta de Fisher. Las asociaciones entre la adherencia de la madre a la administración del hierro y las variables de interés, y entre la administración del hierro y la anemia en el niño se calcularon a través de regresiones logísticas univariadas y multivariadas. El nivel de significación estadística utilizado fue $\alpha=0,05$.

Se obtuvo la aprobación del Comité de Ética y de la Dirección de Atención Primaria de la Salud de la Secretaría de Salud de la Municipalidad de Rosario y el aval del Comité de Ética Independiente del Centro Rosarino de Estudios Perinatales. Se solicitó el consentimiento informado de la madre antes de la inclusión del niño en el estudio.

\section{RESULTADOS}

Se incluyeron 325 pares de madres y niños. Los niños fueron agrupados por edad: menores de 6 meses $(89 / 325 ; 27,4 \%)$, de 6 a 23 meses $(156 / 325 ; 48 \%)$ y de 24 a 42 meses $(80 / 325 ; 24,6 \%)$. Las características generales de la muestra del estudio se presentan en la Tabla 1. En cuanto a la población materna, la edad promedio fue de $26,8 \pm 6,6$ años, $44,3 \%$ refirieron no haber terminado la secundaria y $50,6 \%$ recibían subsidio (asignación universal por hijo). La población infantil del estudio no presentó, en general, problemas de déficit del crecimiento y más de la mitad de los niños habían sido alimentados con pecho en forma exclusiva.

La prevalencia de la anemia a nivel global fue del $40 \%$ (IC $35 \%$ a $45 \%$ ). Al analizar por subgrupo, se observaron diferencias entre los grupos etarios preestablecidos: 19,1\% (IC 10\% a $26 \%$ ) en los menores de 6 meses, $55,8 \%$ (IC 95\% $48 \%$ a $64 \%$ ) en los de 6 a 23 y $32,5 \%$ (IC $22 \%$ a $43 \%)$ en los de 24 meses o más $(p<0,0001)$. En la Figura 1 se muestra la distribución de los valores de hemoglobina para cada uno de estos grupos.

Con respecto al uso de suplementos con hierro por vía oral, el 51,1\% (166/325) de las madres refirieron que su hijo lo había tomado alguna vez. Al desglosar esta información por edades, se observó que la administración de suplementos con hierro en los lactantes menores de 6 meses fue poco frecuente $(6 / 89 ; 6,7 \%)$ en comparación con los de 6 a $23(106 / 156 ; 68 \%)$ y $\geq 24$ meses $(54 / 80$; $67,5 \%)$.

Al considerar el grupo de 6 a 23 meses, se encontró que un tercio de las madres $(48 / 156)$ dijeron estar dando hierro al niño en el momento del ingreso en el estudio: 23 eran niños con anemia $(23 / 87 ; 26,4 \%)$ y $25 \sin$ anemia $(25 / 69 ; 36,2 \%)$. El análisis de regresión logística controlado por la variable "edad del niño" mostró una diferencia significativa $(p=0,025)$.

Por otra parte, las madres de los niños de este grupo etario que nunca habían tomado hierro $(50 / 156 ; 32 \%)$ expresaron que esto no había sido indicado por el médico.

Con el fin de investigar la adherencia a la administración oral del hierro, se consideró a las madres que refirieron haber dado hierro alguna vez, cualquiera que fuera la edad del niño, y que cumplían con los requisitos establecidos para el estudio. El nivel de adherencia en las madres estudiadas ( $n=83$ ) fue del $54 \%$ (IC $43 \%$ a $65 \%$ ). $\mathrm{Al}$ analizar esta variable según el resultado de la prueba rápida de $\mathrm{Hb}$, se observó que en el grupo de niños con anemia la adherencia materna fue del 39\%, mientras que en el grupo 
TABLA 1. Características de las madres y niños del estudio

\begin{tabular}{|c|c|c|c|c|}
\hline & $\begin{array}{c}\text { Muestra total } \\
\quad(\mathrm{n}=325)\end{array}$ & $\begin{array}{c}<6 \text { meses } \\
(n=89)\end{array}$ & $\begin{array}{c}6 \text { a } 23 \text { meses } \\
(n=156)\end{array}$ & $\begin{array}{c}\geq 24 \text { meses } \\
\quad(n=80)\end{array}$ \\
\hline \multicolumn{5}{|l|}{ Características de la población materna } \\
\hline Edad & $26,8 \pm 6,6$ & $26,1 \pm 6,7$ & $26,4 \pm 6,5$ & $28,4 \pm 6,5$ \\
\hline $\begin{array}{l}\text { Nivel de educación } \\
\text { Primaria incompleta } \\
\text { Primaria completa } \\
\text { Secundaria incompleta } \\
\text { Secundaria completa } \\
\text { Nivel terciario }\end{array}$ & $\begin{array}{c}10(3,1 \%) \\
69(21,2 \%) \\
144(44,3 \%) \\
69(21,2 \%) \\
33(10,1 \%)\end{array}$ & $\begin{array}{c}2(2,2 \%) \\
19(21,4 \%) \\
37(41,6 \%) \\
21(23,6 \%) \\
10(11,2 \%)\end{array}$ & $\begin{array}{c}4(2,6 \%) \\
39(25 \%) \\
66(42,3 \%) \\
37(23,7 \%) \\
10(6,4 \%)\end{array}$ & $\begin{array}{c}4(5 \%) \\
11(13,8 \%) \\
41(51,3 \%) \\
11(13,8 \%) \\
13(16,3 \%)\end{array}$ \\
\hline $\begin{array}{l}\text { Características de la población infanti } \\
\text { Niñas } \\
\text { Peso al nacer }<2500 \mathrm{~g} \\
\text { Duración de la lactancia (meses) } \\
\text { Lactancia exclusiva }^{1}\end{array}$ & $\begin{array}{c}155(47,7 \%) \\
29(8,9 \%) \\
9 \pm 7,9 \\
179(55,3 \%)\end{array}$ & $\begin{array}{c}47(52,8 \%) \\
7(7,8 \%) \\
2,2 \pm 1,6 \\
61(68,5 \%)\end{array}$ & $\begin{array}{c}70(44,9 \%) \\
11(7 \%) \\
9,9 \pm 5,9 \\
79(50,6 \%)\end{array}$ & $\begin{array}{c}38(47,5 \%) \\
11(13,8 \%) \\
15,1 \pm 9,5 \\
39(49,4 \%)\end{array}$ \\
\hline $\begin{array}{l}\text { Crecimiento }^{2} \\
\text { Sin déficit } \\
\text { Bajo peso } \\
\text { Baja talla } \\
\text { Emaciación }\end{array}$ & $\begin{array}{c}278(90,6 \%) \\
1(0,3 \%) \\
16(5,2 \%) \\
12(3,9 \%)\end{array}$ & $\begin{array}{c}74(88,1 \%) \\
0(0 \%) \\
6(7,1 \%) \\
4(4,8 \%)\end{array}$ & $\begin{array}{c}133(89,9 \%) \\
1(0,7 \%) \\
8(5,4 \%) \\
6(4,1 \%)\end{array}$ & $\begin{array}{c}71(94,7 \%) \\
0(0 \%) \\
2(2,7 \%) \\
2(2,7 \%)\end{array}$ \\
\hline Anemia & $130(40 \%)$ & $16(19,1 \%)$ & $87(55,8 \%)$ & $26(32,5 \%)$ \\
\hline Subsidio para el niño ${ }^{1}$ & $164(50,6 \%)$ & $37(42 \%)^{1}$ & $79(50,6 \%)$ & $48(60 \%)$ \\
\hline
\end{tabular}

Variable categórica: $\mathrm{n}(\%)$; variable continua: media \pm desviación estándar.

Valor límite para hemoglobina en niños $<6$ meses: 9,5 g/dL y en niños $\geq 6$ meses: $11 \mathrm{~g} / \mathrm{dL}$.

${ }^{1}$ Un dato faltante; ${ }^{2} 18$ datos faltantes.

FIGURA 1. Distribución de los valores de hemoglobina $(\mathrm{g} / \mathrm{dL}$ ) según grupos etarios

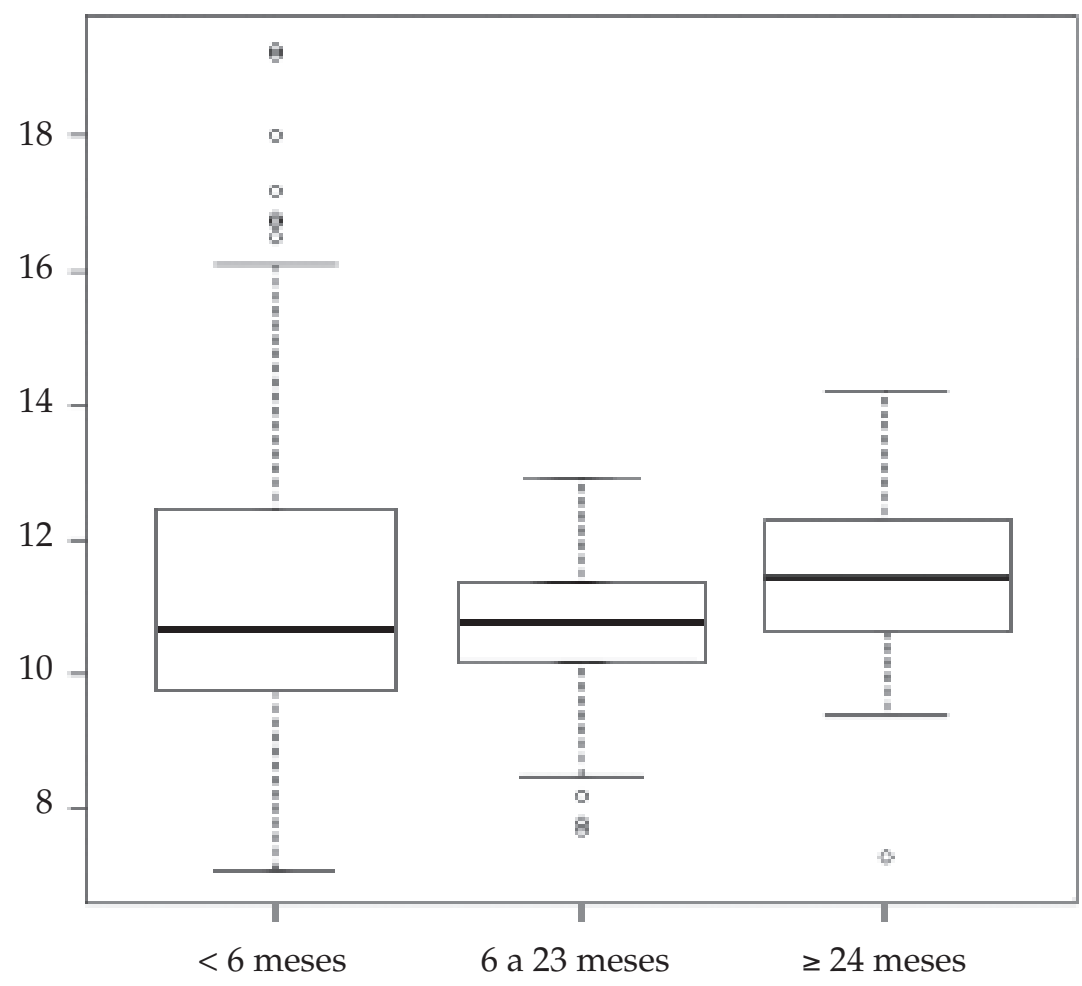


de niños sin anemia fue del 70\% (OR 0,28; IC 0,11 a 0,69$)$. Las razones para la falta de adherencia fueron: intolerancia digestiva $(38 \%)$, olvido $(36 \%)$, decisión personal de la madre $(11 \%)$, falta de entendimiento $(6 \%)$, problemas de acceso al sistema de salud ( $3 \%)$, y otros como alergia o fiebre $(6 \%)$.

Se exploró la potencial asociación entre la adherencia de las madres a la administración del hierro en el niño y las siguientes variables: edad y nivel educativo de la madre (años), antecedente de anemia en el niño expresado por la madre (sí/no), lactancia exclusiva (sí/no), baño con botón o cadena (sí/no) y otorgamiento de subsidio para el niño (sí/no). Las dos últimas variables mencionadas permitieron definir un grupo de familias de nivel social más bajo a pesar de la homogeneidad de la muestra y de no haberse recolectado datos socioeconómicos más específicos.

Se observó un mayor grado de adherencia a la suplementación con hierro en las madres de más edad (OR 1,04; IC 0,97 a 1,11) y con mayor nivel de educación alcanzado (OR 1,14; IC 0,94 a 1,38), aunque estas asociaciones no fueron significativas (Tabla 2).

\section{DISCUSIÓN}

La prevalencia de la anemia en la muestra estudiada fue diferente entre los grupos de edades considerados. En el grupo de lactantes menores de 6 meses fue del 19\%. Este hallazgo podría estar explicado, entre otras razones, por el estado nutricional de las madres y por la baja implementación de la ligadura tardía del cordón umbilical. En el grupo de 6 a 23 meses de edad, más de la mitad de los niños estaban anémicos. Este resultado pone de manifiesto el valor de la profilaxis con hierro a partir del sexto mes de vida como estrategia de salud pública.

Existen estudios que aportan datos concretos sobre los factores que podrían explicar la persistente alta prevalencia de la anemia en las poblaciones infantiles de nuestro país. ${ }^{16,17,25}$ De forma inédita, nuestro trabajo ha abordado el tema de la adherencia a la administración del hierro teniendo en cuenta las respuestas de las madres que llevan a sus hijos a la consulta de pediatría ambulatoria en la red de atención pública. En este contexto, al menos una madre de cada dos no cumplió con la indicación del médico pediatra.

El motivo más frecuente para la falta de adherencia fue "la intolerancia digestiva". En relación con este efecto adverso, los resultados del estudio de Donato y cols. ${ }^{26}$ no mostraron diferencias al comparar el sulfato ferroso con el hidróxido férrico polimaltosado. De aquí se desprende la importancia de alertar oportunamente sobre los posibles efectos indeseables del hierro, explicar que existen otras alternativas de tratamiento en caso de rechazo a la medicación y enfatizar sobre el valor del cumplimiento del esquema de administración del hierro para lograr el efecto buscado.

La segunda causa referida por las madres fue "el olvido". Esto podría estar relacionado con la poca importancia que tiene para ellas la administración de gotas de hierro en un niño que, por lo general, goza de buena salud y no presenta problemas de bajo peso. La concepción de la madre sobre lo que significa la anemia se potencia ante su desconocimiento acerca de los efectos perjudiciales sobre el crecimiento y desarrollo del niño, y la gran heterogeneidad en el manejo clínico de la anemia ferropénica. ${ }^{16,17}$

En este estudio pueden considerarse varias limitaciones. Se presume que la mayoría de los casos de anemia son causados por déficit de hierro. ${ }^{2,4,11} \mathrm{La}$ adherencia a la administración del hierro por vía oral se determinó a partir de las respuestas de las madres, lo cual podría

TABLA 2. Variables de asociación para adherencia de las madres a la administración oral de hierro en el niño (n=83)

\begin{tabular}{lcr} 
Variable de interés & \multicolumn{2}{c}{ Análisis de regresión logística } \\
\cline { 2 - 3 } & $\begin{array}{c}\text { Univariado } \\
\text { OR (IC 95\%) }\end{array}$ & $\begin{array}{r}\text { Mrivariado }(\text { IC 95\%) } \\
\text { Oducación materna (años) }\end{array}$ \\
\hline Edad materna (años) & $1,13(0,95-1,36)$ & $1,04(0,97-1,38)$ \\
Antecedente de anemia en el niño & $1,03(0,97-1,10)$ & $1,64(0,65-4,15)$ \\
Lactancia exclusiva & $1,60(0,67-3,84)$ & $0,72(0,29-1,79)$ \\
Baño con botón o cadena & $0,62(0,26-1,50)$ & $0,86(0,27-2,74)$ \\
Subsidio familiar & $0,81(0,27-2,42)$ & $1,77(0,68-4,63)$ \\
\hline
\end{tabular}


introducir sesgos, como el del recuerdo y el de la conveniencia. Con respecto a las variables estudiadas en cuanto a la adherencia de las madres, la falta de significación estadística podría explicarse por la falta real de asociación, ${ }^{27,28}$ aunque también puede deberse a la homogeneidad en términos sociodemográficos de las familias incluidas en el estudio ya que, por ejemplo, solo se incluyeron familias usuarias del sistema de salud público, o al pequeño tamaño de la muestra.

Algunos estudios sugieren que el conocimiento específico (signos, síntomas, efectos adversos, etc.) sobre una enfermedad es un factor potencialmente asociado al grado de adherencia al tratamiento. ${ }^{29-31}$ No obstante, esto no fue explorado en nuestro estudio.

A pesar del carácter local de los hallazgos, emerge la necesidad de desarrollar nuevas líneas de investigación tendientes a identificar los factores (de tipo cultural, socioeconómico, calidad de atención médica, etc.) que estarían interfiriendo en la adherencia de las madres y el impacto de la suplementación con hierro por vía oral en la población infantil.

\section{CONCLUSIONES}

En comparación con los datos disponibles a nivel nacional, la prevalencia de la anemia en la muestra de niños del estudio fue alta. Se observó menor adherencia de las madres a la administración del hierro en el grupo de niños con anemia. No se encontró asociación entre las variables estudiadas y la adherencia materna.

\section{Agradecimientos}

Nuestro reconocimiento a las madres que aceptaron participar en el estudio. Agradecemos especialmente a la Lic. Virginia Lencinas y a la Dra. Daniela Roldán, ya que sin su colaboración este trabajo no hubiera sido posible, y al profesor Eduardo Cuestas por sus comentarios sobre el manuscrito. Nuestra gratitud al Instituto de Salud Global de Duke y al Centro Rosarino de Estudios Perinatales por el apoyo brindado.

\section{BIBLIOGRAFÍA}

1. Worldwide prevalence of anaemia 1993-2005: WHO Global Database on Anaemia. [Acceso: 14 de noviembre de 2012] Disponible en: http://whqlibdoc.who.int/ publications/2008/9789241596657_eng.pdf.

2. WHO,UNICEF,UNU.Iron deficiency anaemia: assessment, prevention, and control. A guide for programmemanagers. Geneva, Switzerland: World Health Organization; 2001. [Consulta: 14 de noviembre de 2012]. Disponible en: http://www.who.int/nutrition/publications/en/ida assessment_prevention_control.pdf.

3. Booth IW, Aukett MA. Iron deficiency anaemia in infancy and early childhood. Arch Dis Child 1997;76(6):549-54.

4. Haemoglobin concentrations for the diagnosis of anaemia and assessment of severity. WHO/NMH/NHD/ MNM/11.1 [Consulta:30 denoviembre de2012]. Disponible en:http://www.who.int/vmnis/indicators/haemoglobin. pdf.

5. Domellöf M, Dewey KG, Lönnerdal B, Cohen RJ, Hermell $\mathrm{O}$. The diagnostic criteria for iron deficiency in infants should be reevaluated. J Nutr 2002;132(12):3680-6.

6. Garcia MT, Granado FS, Cardoso MA. Alimentação complementar e estado nutricional de crianças menores de dois anos atendidas no Programa Saúde da Família em Acrelândia, Acre, Amazônia Ocidental Brasileira. Cad Saúde Pública 2011;27(2):305-16.

7. Netto MP, Priore SE, Sant' Ana HM, Peluzio Mdo C, et al. Prevalence and factors associated to anemia and iron deficiency in 18-to 24-month old infants. Arch Latinoam Nutr 2006;56(3):229-36.

8. Bortolini GA, Vitolo MR. Relationship between iron deficiency and anemia in children younger than 4 years. J Pediatr (Rio J) 2010;86(6):488-92.

9. Shibukawa AF, Silva EM, Ichiki WA, Strufaldi MW, Puccini RFl. Prophylaxis for iron deficiency anemia using ferrous sulfate among infants followed up at a primary healthcare unit in the municipality of Embu-SP (2003/2004). Sao Paulo Med J 2008;126(2):96-101.

10. Hadler MCCM, Yara J, Sigulem DM. Anemia do lactente: etiologia e prevalência. J Pediatr (Rio J) 2002;78(4):321-6.

11. Durán P, Mangialavoria G, Biglieria A, Kogana L, Abeyá Gilardon E. Estudio descriptivo de la situación nutricional en niños de 6-72 meses de la República Argentina. Resultados de la Encuesta Nacional de Nutrición y Salud (ENNyS). Arch Argent Pediatr 2009;107(5):397-404.

12. Lineamientos de prevención y tratamiento de la anemia en la población materno infantil. [Consulta: 4 de noviembre de 2012]. Disponible en: http://www.ms.gba.gov.ar/sitios / seguropublico/files/2012/03/Lineamientos-anemia.pdf.

13. Ceriani Cernadas JM, Carroli G, Pellegrini L, Ferreira M, et al. Efecto del clampeo demorado del cordón umbilical en la ferritina sérica a los seis meses de vida. Estudio clínico controlado aleatorizado. Arch Argent Pediatr 2010;108 (3):201-8,

14. Comité Nacional de Hematología. Sociedad Argentina de Pediatría. Anemia Ferropénica. Guía de diagnóstico y tratamiento. Arch Argent Pediatr 2009;107(4):353-61.

15. Bernztein R. El hierro, la anemia, y el uso racional en el Programa REMEDIAR. Atención Primaria de la Salud. Boletín PROAPS Remediar 2003;1(4):4-7.

16. Bernztein R, Drake I. Subprescripción de hierro y variabilidad en el primer nivel de atención público de la Argentina. Arch Argent Pediatr 2008;106(4):320-7.

17. Sammartino G. Representaciones culturales acerca de la anemia y la suplementación con hierro. Arch Argent Pediatr 2010;108(5):401-8.

18. Galloway R, Mc Guire J. Determinants of compliance with iron supplementation: supplies, side effects, or psychology? Soc Sci Med 1994;39(3):381-90.

19. Lutter CK. Iron deficiency in young children in low-income countries and new approaches for its prevention. J Nutr 2008;138(12):2523-8.

20. Ip H, Hyder SM, Haseen F, Rahman M, Zlotkin SH. Improved adherence and anaemia cure rates with flexible administration of micronutrient Sprinkles: a new public health approach to anaemia control. Eur J Clin Nutr 2009;63(2):165-72. 
21. Geltman PL, Hironaka LK, Mehta SD, Padilla P, et al. Iron supplementation of low-income infants: a randomized clinical trial of adherence with ferrous fumarate sprinkles versus ferrous sulfate drops. J Pediatr 2009;154(5):738-43.

22. Development of an international guide to monitor and support child development (Project number: 5R01HD057834-02). Research Portfolio Online Reporting Tools (RePORT), National Institutes of Health, U. S. Department of Health and Human Services. [Consulta: 17 de agosto de 2012]. Disponible en: http:/ / projectreporter. nih.gov / project_info_description.cfm?aid $=8118246 \&$ icd $\mathrm{e}=12506745 \&$ ddparam $=\&$ ddvalue $=\& d d s u b=\& c r=1 \& \mathrm{csb}$ $=$ default\&cs $=$ ASC

23. Muñoz M, Romero A, Gómez JF, Manteca A, et al. Utility of point-of-carehaemoglobin measurement in the HemoCue-B haemoglobin for the initial diagnosis of anaemia. Clin Lab Haematol 2005;27(2):99-104.

24. SAS. SAS, the power to know. SAS Institute Inc.

25. IanicelliJC, Varea A, Falivene M, Disalvo L, et al. Prevalencia de anemia en lactantes menores de 6 meses asistidos en un centro de atención primaria de la ciudad de La Plata. Arch Argent Pediatr 2012;110(2):120-5.
26. Donato H, Rapetti MC,Morán L, Cavo M.Hidróxido férrico polimaltosado contra sulfato ferroso en el tratamiento de la anemia ferropénica: estudio prospectivo aleatorizado. Arch Argent Pediatr 2007;105(6): 491-7.

27. Lacerte P, Pradipasen M, Temcharoen P, Imamee N, Vorapongsathorn T. Determinants of adherence to iron/ folate supplementation during pregnancy in two provinces in Cambodia. Asia Pac J Public Health 2011;23(3):315-23.

28. Dairo MD, Lawoyin TO. Demographic factors determining compliance to iron supplementation in pregnancy in Oyo State, Nigeria. Niger J Med 2006;15(3):241-4.

29. Janakan N,SeneviratneR. Factors contributing to medication noncompliance of newly diagnosed smear-positive pulmonary tuberculosis patients in the district of Colombo, Sri Lanka. Asia Pac J Public Health 2008;20(3):214-23.

30. Bilenko N, Yehiel M, Inbar Y, Gazala E. The association between anemia in infants, and maternal knowledge and adherence to iron supplementation in southern Israel. Isr Med Assoc J 2007;9(7):521-4.

31. Hironaka LK, Paasche-Orlow MK, Young RL, Bauchner H, Geltman PL. Caregiver health literacy and adherence to a daily multi-vitamin with iron regimen in infants. Patient Educ Couns 2009;75(3):376-80. 